\title{
Research Paper \\ Patterns of Muscle Activity Measurement in Male Students With Forward Head Posture Compared to Healthy Peers During Running
}

\author{
${ }^{*}$ Amirali Jafarnezhadgero ${ }^{1} \oplus$, Saeideh HeshmatiZadeh ${ }^{2}$, Somaye Salahi-Movasagh ${ }^{3}$, Farzaneh Saki ${ }^{4}$
}

1. Assistant Professor of Sport Biomechanics, Department of Physical Education and Sport Sciences, Faculty of Educational Sciences and Psychology, University of Mohaghegh Ardabili, Ardabil, Iran.

2. Master of Science Student of Sport Biomechanics, Department of Physical Education and Sport Sciences, Faculty of Education Sciences and Psychology, University of Mohaghegh Ardabili, Ardabil, Iran.

3. Master of Science Student of Sport Injuries, Department of Sport Injuries, Faculty of Physical Education and Sport Sciences, University of Allameh Tabatabaii, Tehran, Iran.

4. Assistant Professor of of Sport Injuries, Department of Sport Injuries, Faculty of Physical Education and Sport Sciences, Bu Ali Sina University, Hamadan, Iran.

Citation: Jafarnezhadgero A, \& Heshmatizadeh S. [The Timing Pattern of Selected Muscles in Male Children With Forward Head Posture Compared to Healthy Control Ones During Running (Persian)]. Journal of Sport Biomechanics. 2019; 5(1):38-49. https://doi.org/10.32598/biomechanics.5.1.4

doi https://doi.org/10.32598/biomechanics.5.1.4

Key words:

Running, Forward

head posture, Muscu-

lar activity timing

\section{A B STRACT}

Objective The aim of this study was to evaluate the muscular activity timing during running in male children with forward head posture compared to healthy control ones.

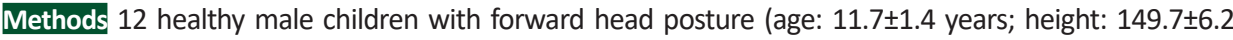
$\mathrm{cm}$; Mass: $38.0 \pm 4.7 \mathrm{~kg}$ ) and 16 healthy male children (age: $11.8 \pm 1.5$ years; height: $148.2 \pm 6.6 \mathrm{~cm}$; mass: $39.6 \pm 0.4 \mathrm{~kg}$ ) were volunteered to participate in this study. The timing of muscular activity for erector spinae (cervical region), sternocleidomastoid, serratus anterior, upper and lower trapezius muscles of both right and left sides were evaluated during barefoot running. Muscular activity was recorded with a 16-chanels electromyography system. Independent sample t-test was used for statistical analysis.

Results Finding did not demonstrate any significant differences in onset and offset of muscular activity during walking between both groups $(P>0.05)$. The time duration of right erector spinae muscle in the healthy group was greater than that forward head group about $41 \mathrm{~ms}(\mathrm{P}=0.024)$. Also, the time duration of left sternocleidomasteoid in the healthy group was longer than that in the forward head group by 64 $\mathrm{ms}(\mathrm{P}=0.037)$.

Conclusion In sum, these results could be used in rehabilitation protocols for children with forward head posture in clinical settings.

\section{Extended Abstract}

\section{Introduction}

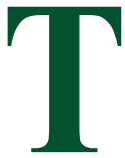

he head makes up $6 \%$ of the total body weight; the spine, neck, and other joints connect to the muscles through the kinematic chain. Keeping the cervical spine in a fixed position for a long time could alter head posture. It could eventually lead to a weakening of the body, i.e. a Forward Head Posture (FHP), which is a deviation from the neutral or normal position [1, 2]. Normal position is defined as a line of gravity that passes through the external auditory canal of the spine and neck and from the front to the thoracic vertebrae [3]. This condition must be morphologically ac-

\section{* Corresponding Author:}

Amirali Jafarnezhadgero, PhD.

Address: Department of Physical Education and Sport Sciences, Faculty of Educational Sciences and Psychology, University of Mohaghegh Ardabili, Ardabil, Iran.

Tel: +98 (910) 5146214

E-mail: amiralijafarnezhad@gmail.com 
ceptable. Besides, one must be able to maintain a state of discomfort and painlessness.

The FHP is among the most frequent abnormalities in patients with cervical disorders [4]. Studies have estimated its prevalence to be $66 \%-80 \%$ (5). The FHP refers to the anterior position of the head relative to the trunk concerning a vertical reference line $[7,6]$. However, the pattern of muscle activity measurement in people with anatomical abnormalities, especially in those with FHP complications during running, has rarely been studied.

The present study aimed to evaluate the measurement pattern of selective trunk and cervical muscles activity in high school students with anatomical abnormalities, compared to their healthy peers. The measurements were conducted during the onset and time of activity and muscle activity decline in running. Evaluating muscle activity based on the measurement variables of the mentioned cases can provide useful information for rehabilitation in these individuals.

\section{Participants and Methods}

This was a cross-sectional study. It was conducted using information from previous studies to estimate the sample size of $\geq 24$ people to obtain statistical power of 0.8 at a significant level of $\mathrm{P}<0.05$ [21]. The study subjects included 16 healthy boys and 12 boys with FHP. The study participants' head angle was measured by goniometer.
Using a 16-channel Wireless Electromyography (EMG) (BTS FREEEMG300, BTS Bioengineering, Italy) with bipolar disposable surface electrodes, erector spinae (cervical part), sternocleidomastoid, upper trapezoid, anterior indentation, and upper and lower trapezius (right and left) were recorded while running. The center-to-center electrodes distance was equal to $2 \mathrm{~cm} \mathrm{[23].}$

The electrical signals were recorded at a sampling frequency of $1200 \mathrm{~Hz}$. Low-pass filter $500 \mathrm{~Hz}$ and the highpass filter $10 \mathrm{~Hz}$ and $60 \mathrm{~Hz}$ non-pass filter (to remove AC power noise) were used to filter raw Electromyography (EMG) data [26]. The machine's gain was set at 1000 .

\section{Results}

The obtained findings suggested no statistically significant difference between the two groups in the onset of the muscle activity (erector spinae, sternocleidomastoid, upper and lower trapezius, left and right anterior indentation) $(\mathrm{P}<0.05)$. The effect size values were in all cases, from small to medium (Table 1).

Furthermore, there was no statistically significant difference between the two groups in terms of the ending phase of muscle activity (erector spinae, sternocleidomastoid, upper and lower trapezoid, left and right anterior indentation) $(\mathrm{P}<0.05)$. The effect size values were small to medium in all cases.

Table 1. The time of the muscle activity onset during the running phase in two groups of healthy boys and with FHP complications

\begin{tabular}{|c|c|c|c|c|c|}
\hline \multirow{2}{*}{ Side } & \multirow{2}{*}{ Muscle } & \multicolumn{2}{|c|}{ Mean \pm SD } & \multirow{2}{*}{ Significant Size } & \multirow{2}{*}{ Effect Level } \\
\hline & & Healthy & FHP & & \\
\hline \multirow{5}{*}{ Wright } & Erector spinae & $-149.1 \pm 267.3$ & $-99.1 \pm 33.8$ & 0.527 & 0.332 \\
\hline & Sternocleidomastoid & $-95.8 \pm 129.4$ & $-109.2 \pm 151.2$ & 0.802 & 0.09 \\
\hline & Upper trapezoid & $-87.3 \pm 66.4$ & $-135.4 \pm 115.2$ & 0.175 & 0.264 \\
\hline & Anterior indentation & $-94.1 \pm 131.3$ & $-61.8 \pm 16.7$ & 0.345 & 0.436 \\
\hline & Lower trapezoid & $-90.9 \pm 68.8$ & $-194.8 \pm 344.8$ & 0.248 & 0.502 \\
\hline \multirow{5}{*}{ Left } & Erector spinae & $-93.2 \pm 37.6$ & $-63.9 \pm 39.2$ & 0.223 & 0.673 \\
\hline & Sternocleidomastoid & $-91.9 \pm 111.4$ & $-75.3 \pm 22.6$ & 0.616 & 0.247 \\
\hline & Upper trapezoid & $-75.6 \pm 121.1$ & $-87.7 \pm 32.8$ & 0.741 & 0.157 \\
\hline & Anterior indentation & $-112.9 \pm 123.6$ & $-79.6 \pm 37.8$ & 0.377 & 0.206 \\
\hline & Lower trapezoid & $-71.7 \pm 18.3$ & $-73.5 \pm 27.9$ & 0.848 & 0.077 \\
\hline
\end{tabular}

*Significance level: $\mathrm{P}<0.05$ 


\section{Discussion}

The present study evaluated the timing pattern of trunk and neck muscles in male students with FHP disorder, compared to their healthy peers during running. Besides, there was no statistically significant difference between the two groups at the beginning and end of the selected muscle activity.

A study compared shoulder muscle memory in patients with idiopathic neck pain and a healthy group. The relevant data indicated that the anterior indentation muscle delayed the onset and duration of shorter activity during dynamic movements [27]. They also found no significant difference in the onset and duration of trapezius muscle activity between patients with idiopathic neck pain and the healthy group [27]. Cools et al. reported no significant difference in the time of activity in the upper trapezius muscle between the controls and the group with shoulder pain syndrome during running [19]. Some researchers argued that people with musculoskeletal disorders have more muscle activity than healthy individuals, especially in the trapezius muscles $[28,29]$. These changes in neck posture can lead to abnormal movement patterns in this area [12]. Some studies have documented that changes in muscle activity were associated with changes in muscle frequency [30].

\section{Conclusion}

The onset and end of the selective trunk and neck muscle activity in the running phase demonstrated no statistically significant difference between the healthy individuals and those with FHP disorder. However, the activity duration of the erector spinae muscles of the right side and sternocleidomastoid of the left side were greater in the healthy group, compared to the FHP group. Additionally, more activity in these muscles was attributed to their weaker muscles. These findings could be used in the rehabilitation of people with FHP in therapeutic settings.

\section{Ethical Considerations}

\section{Compliance with ethical guidelines}

All ethical principles were considered in this article. The participants were informed about the purpose of the research and its implementation stages; they were also assured about the confidentiality of their information; Moreover, They were allowed to leave the study whenever they wish, and if desired, the results of the research would be available to them.

\section{Funding}

This research did not receive any specific grant from funding agencies in the public, commercial, or not-forprofit sectors.

\section{Authors' contributions}

All authors contributed equally in preparing all parts of the research.

\section{Conflicts of interest}

The authors declared no conflict of interest. 


\title{
الكَوى زمانبندى فعاليت عضلات منتخب در دانش آموزان يسر داراى عارضه سربهجلو در مقايسه با همسالان سالم طى دويدى فيدن
}

\author{
"اميرعلى جعفرنزادكرو' [ن، سعيده حشمتى زاده ، سميه صلاحى موثق"، فرزانه ساكى"
}

ا. استاديار بيومكانيك ورزشى، كروه تربيت بدنى و علوم ورزشى، دانشكده علوم تربيتى و روانشئاسى، دانشُكاه هحقق اردبيلى، اردبيل، ايران.

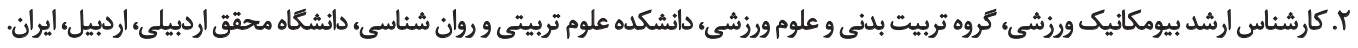

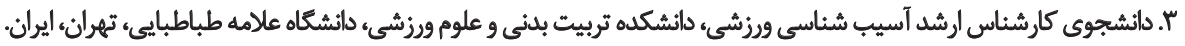

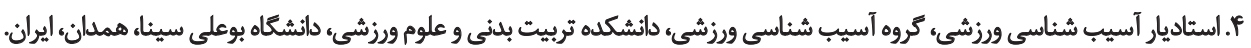

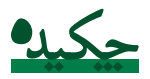

هدف هدف از اين مطالعه بررسى الكوى زمانبئدى فعاليت عضلات مئتخب طي دويدن در دانشيآموزان يسران داراي عارضه سربهجلو

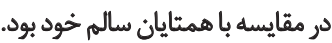

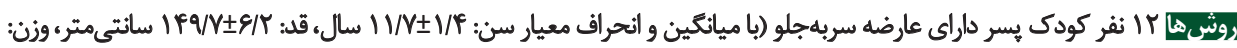

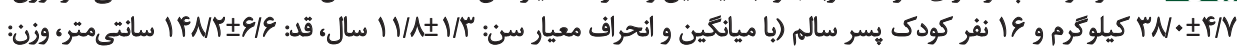

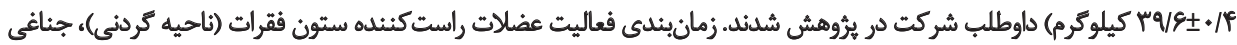

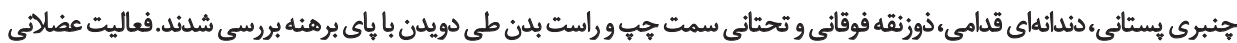

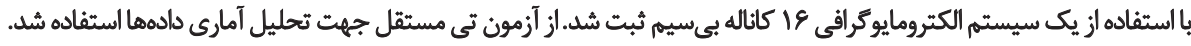

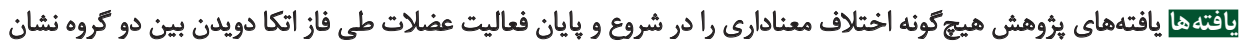

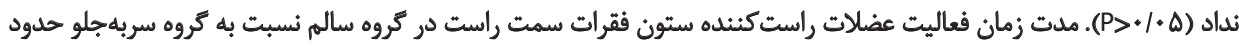

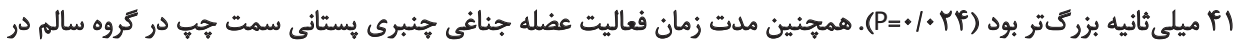

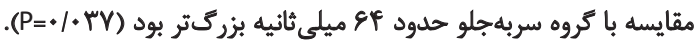

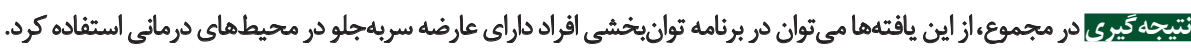

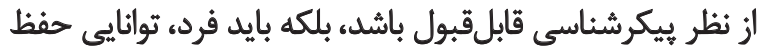

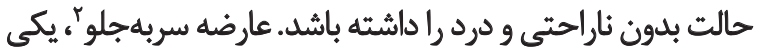

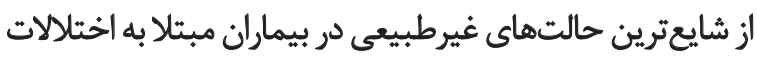

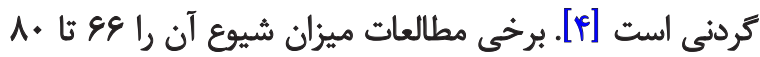

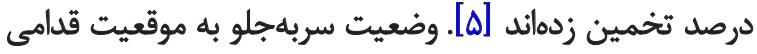

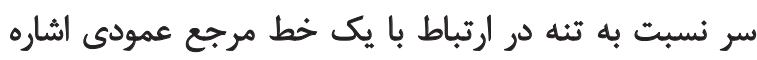

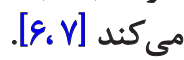

عملكرد اصلى مهرهماى كردنى، حفظ سر در برابر نيروى

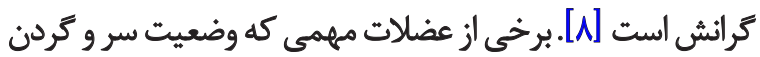

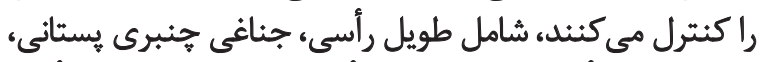

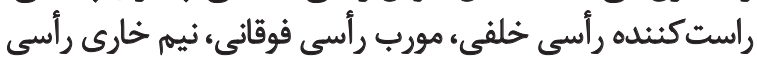

سر شش درصد از وزن كل بلن را تشكيل مى دهد كه ستون

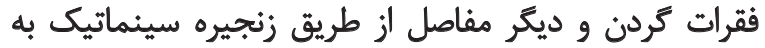

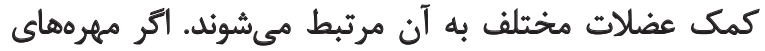

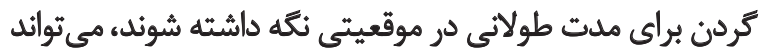

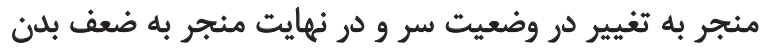

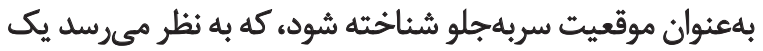

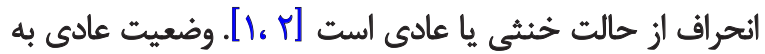

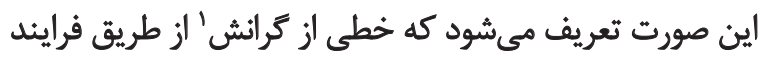

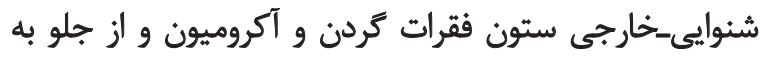

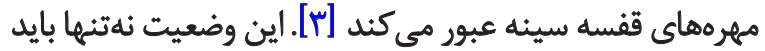

1. Line of gravity (LOG)

\section{-}

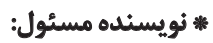

دكتر امير على جعفرنثرادئرو

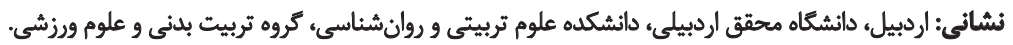

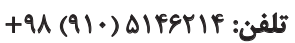
يست الكترونيكي: تلئ 
شدهاند. هدف از ئوهش حاضر بررسى الكوى زمانبيندى

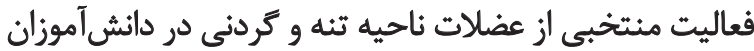

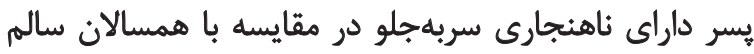

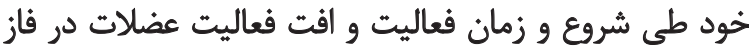

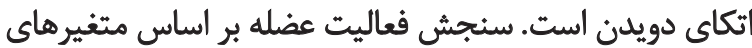

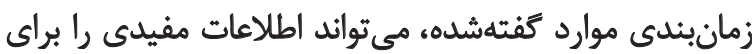
توانبخشى در اين افراد فراهم آورد.

روش ترشناسى

آزمودنىها

اين ثروهش از نوع مقطعى عرضى است كه با استفاده از

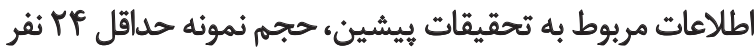

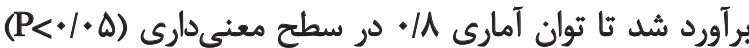

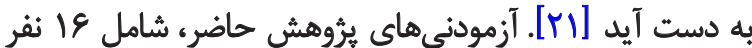

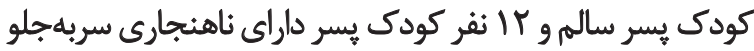

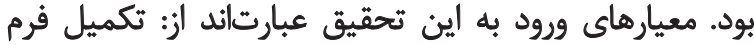

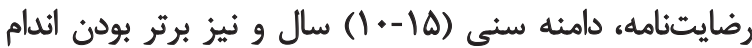

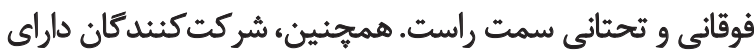

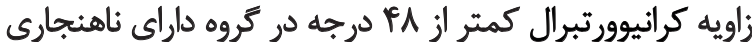

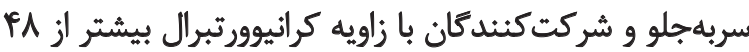
درجه در كروه سالم قرار ترفتند.

ميانكين و انحراف معيار زاويه كرانيوورتبرال در افراد داراي

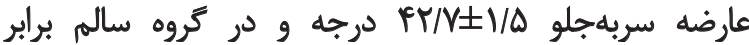

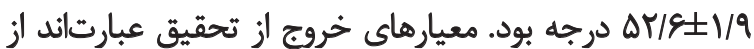

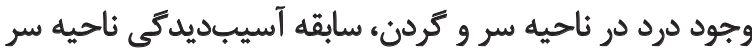

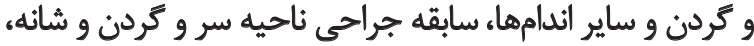

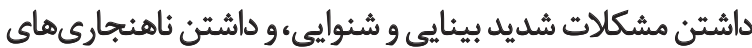

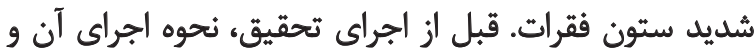

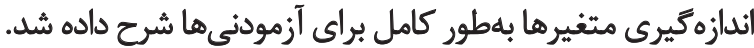

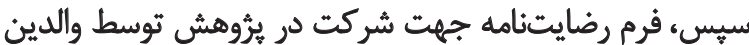

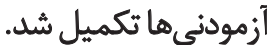

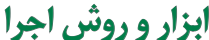

روش اندازهكيرى زاويه سر به وسيله كونيامتر اندازهكيرى شد،

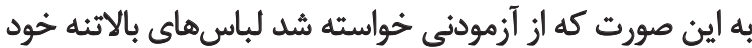

مى شود [9]. عارضه سربهجلو منجر به افزايش لوردوز كردنى،

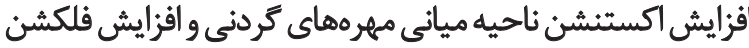

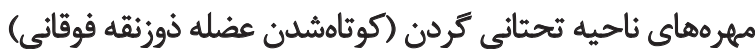

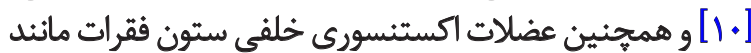

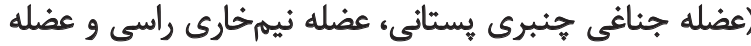

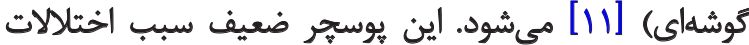

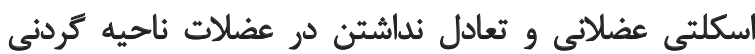

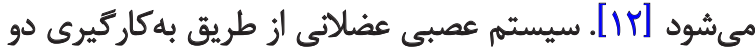

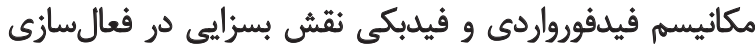

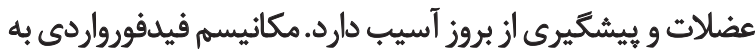

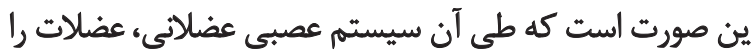

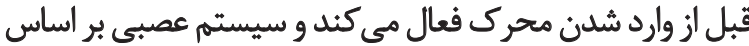

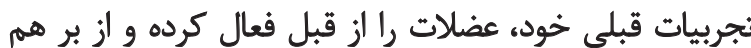

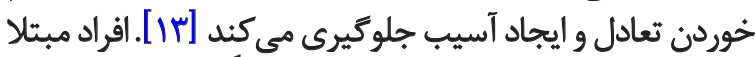

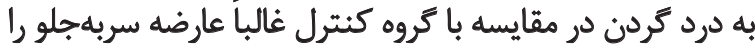

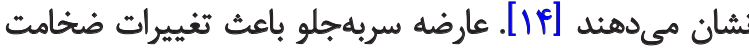

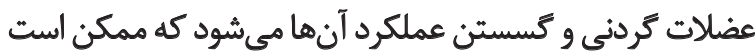

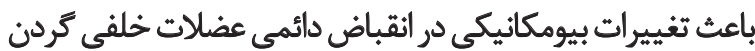
و كشتاور فلكسور اضافى در آنها شود [1 إن].

يارامترهاى مختلفى را مىتوان از سيكنال الكترومايوكرافى

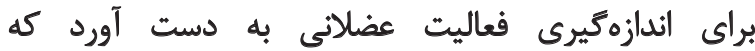

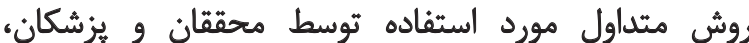

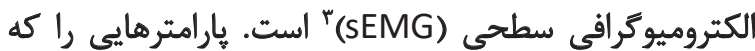

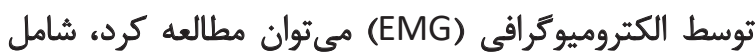

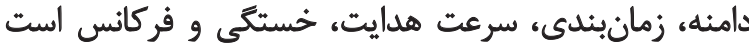

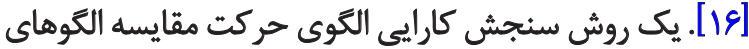
فعال سازي عضله (MAPs) در كنترل و آزمايش آز آزمودنى هاست

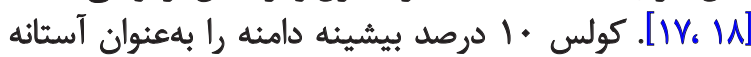

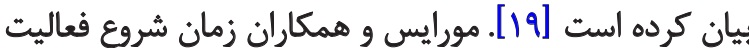

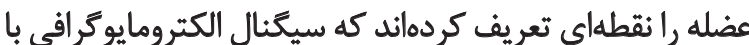

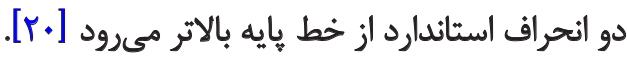
با وجود اين، الكوى زمانبندى فعاليت عضلانى در افراد

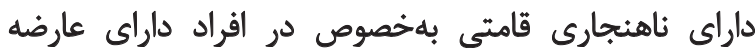

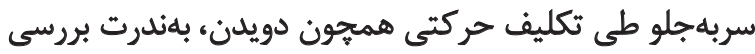
3. Electromyography

جدول ا.ويرّكي هاي جمعيتشناختي آزموننيها

\begin{tabular}{|c|c|c|c|c|}
\hline \multicolumn{3}{|c|}{ ميانكين +انحر اق معيار } & \multirow{2}{*}{ تعداد } & \multirow{2}{*}{ آزمودنى } \\
\hline وزن (كيلوكرم) & قد (سانتى مثر) & سن (سال) & & \\
\hline$r q / \mathscr{q} \pm / f$ & IFNT $\pm \& / \&$ & $11 / \lambda \pm 1 / \pi$ & 18 & كروه سالم \\
\hline$Y N P \pm \cdot N$ & $\mid e q / N \pm g / r$ & $11 / N \pm 1 /{ }^{\circ}$ & 18 & كروه سربهجلو \\
\hline
\end{tabular}

مجله بيومكانيك ولنشـ 


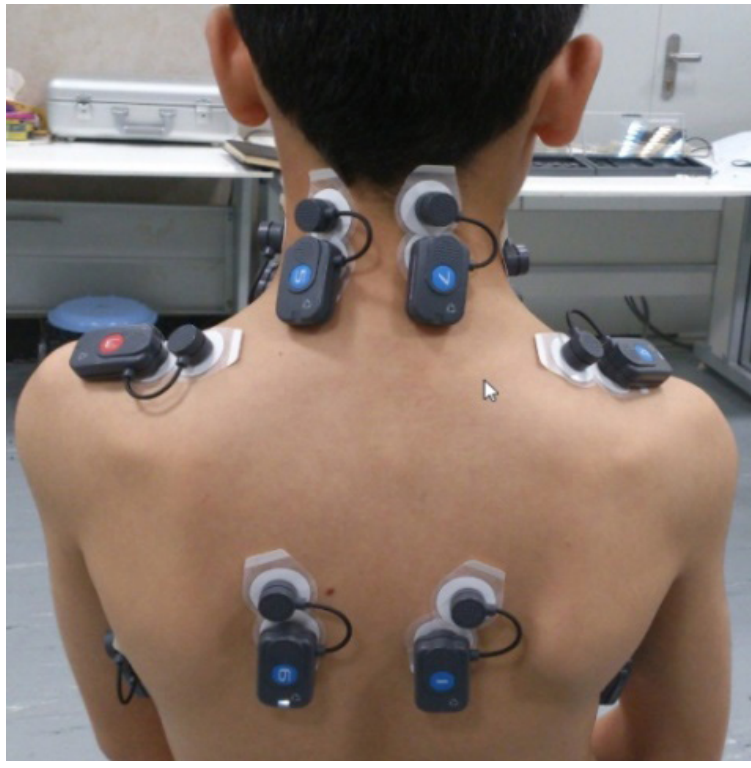

مجله بيومكانيك وزنث

تصوير T. محل نصب الكترودها بر روى عضلات منتخب

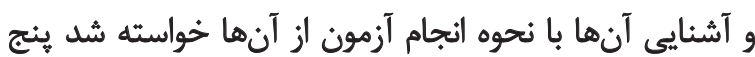

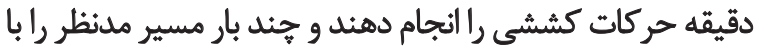

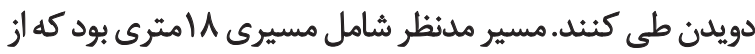

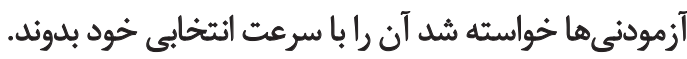

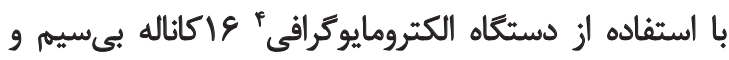

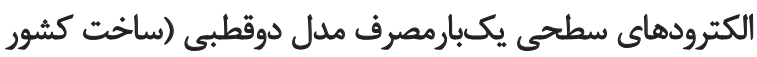

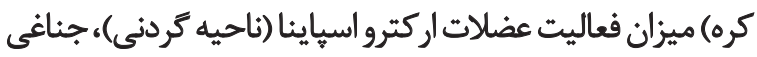

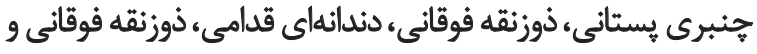

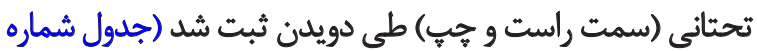

4. BTS FREE EMG300, BTS Bioengeeri, Italy

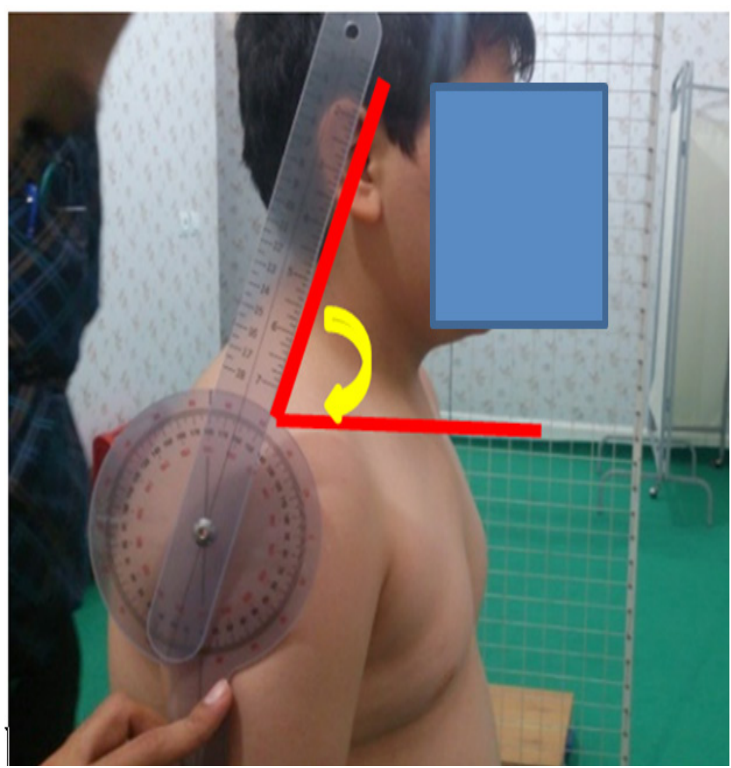

مجله بيومكانيك ولنش

تصوير ا. روش اندازهيرى زاويه سربهجلو با استفاده از كونيامتر

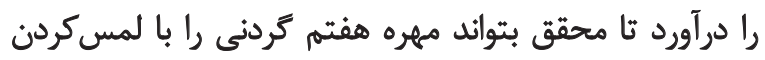

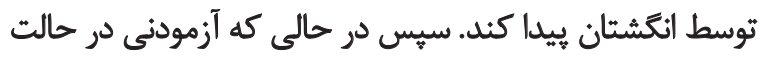

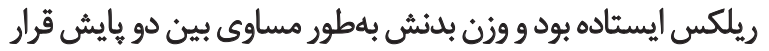

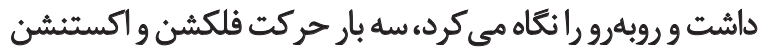

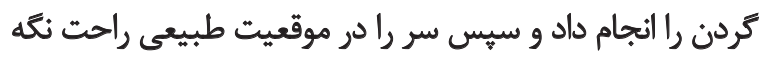

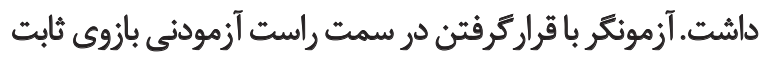

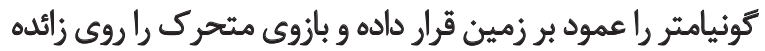

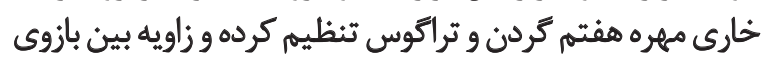

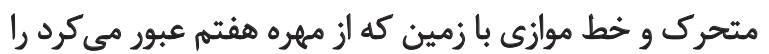

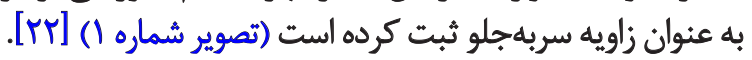
بعد از اندازهيرى وزن و قد آزمودنىها (جدول شماره ()

جدول r. موقعيت قراردادن الكترودها بر روى هر عضله

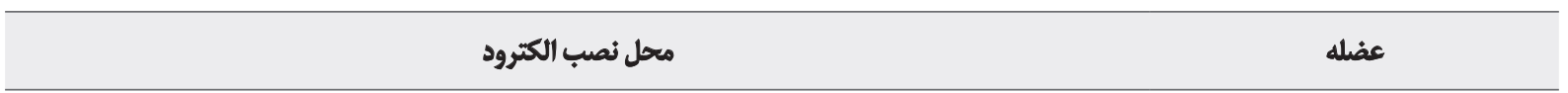

روى سر جناغى و بر روى برجستهترين ناحيه آن، در يكسوم تحتانى ابتدا و انتهاى عضله (الز زائده ماستوئيد ثا بريدكى جrF] جناغى

جناغى جنبرى بيستانى

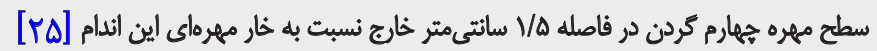

راست كنثله ستون فقرات (ناحيه كردئى)

هحل قراركيرى الكترودها در نيمه خطى است كه از أكروميون به ستون فقرات در مهره هفتم كردئى (CV) تصور مى شود.

ذوزئقه فوقانىى

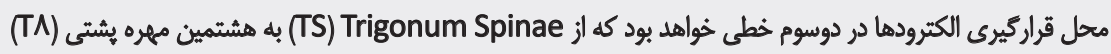
تصور مي شُود.

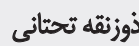

به صورت عمودى در راستاى خط زيربغلى در سطح دندهاي عو 1

دنداثهاي قدامى

مجله بيومكانيك ورنشث 


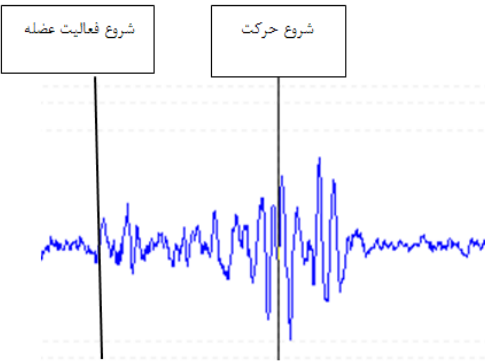

مجله بيومكانيك وزنش

براى بررسى طبيعىبودن توزيع دادهها از آزمون شاييرو-ويلك

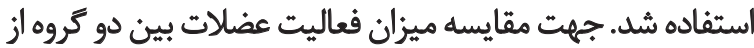

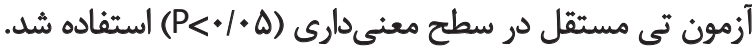

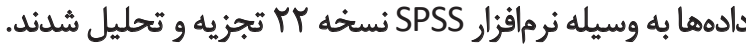

ثتايج

يافتههاى يروهش حاضر نشان داد كه در شروع فعاليت عضلات

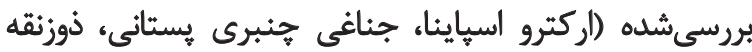

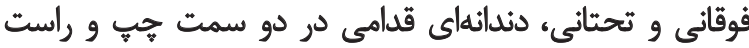

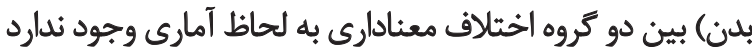

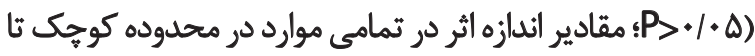

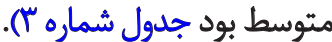

يافتههاي يُروهش حاضر نشان داد كه در مقادير يايان فعاليت

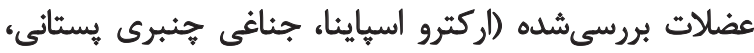

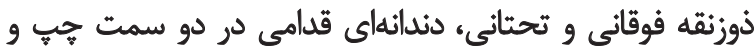

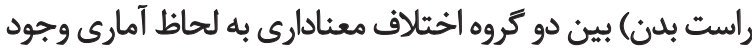

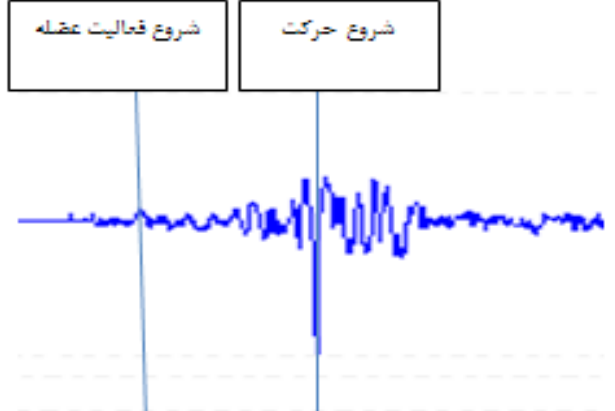

تصوير ب. زمان شروع حركت و زمان شروع به فعاليث الكترومايوكرافى

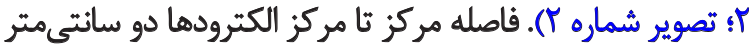

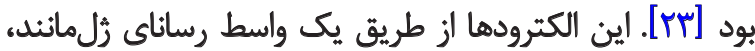

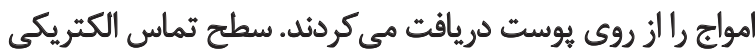

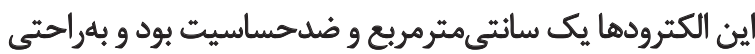

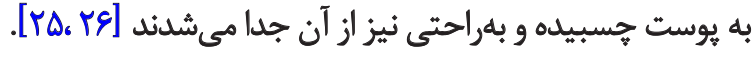

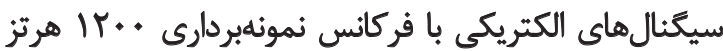

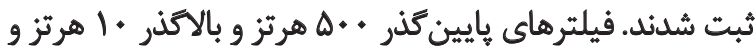

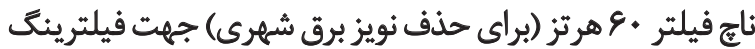

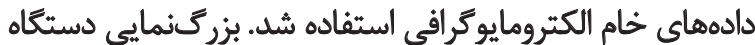

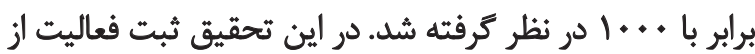

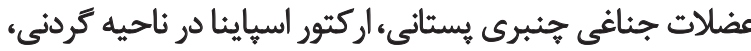

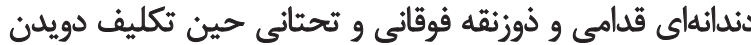

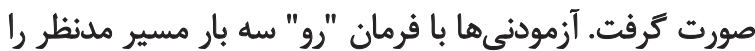

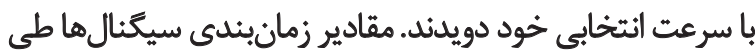

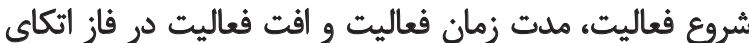
لدويدن محاسبه شد (تصوير شماره "َ).

جدول "r. زمان شروع فعاليت عضلات طى فاز اتكاى دويدن در دو گروه سالم و داراى عارضه سربهجلو

\begin{tabular}{|c|c|c|c|c|c|}
\hline \multirow{2}{*}{ اندازه اثر } & \multirow{2}{*}{ سطح معنىدارى } & \multicolumn{2}{|c|}{ ميانكين } & \multirow{2}{*}{ عضله } & \multirow{2}{*}{ سمت } \\
\hline & & ئروه سربه جلو & كروه سالم & & \\
\hline . & . larr & $-99 / \pm m / 1$ & $-\mid$ IF/reV $\pm / / r$ & اركترو السيايثا & \\
\hline .1 .9 & $M \cdot r$ & $-1 \cdot 9 / r \pm 101 / r$ & $-q \Delta / / Y q \pm N F$ & جناغي هنبرى يستانى & \\
\hline - MeP &.$/ 1 V D$ & $-1 r \Delta / t \pm \| 11 / / r$ & $-A V / e q \pm r / \%$ & ذوزنقهه فوقائي & | - ماسث \\
\hline . Mtre &.$/ M P a$ & $-\varepsilon / / A \pm I E / N$ & $-q p / / r^{m} \pm 1 / r$ & دئداثهاي قدامى & \\
\hline $.10 \cdot r$ & . MTA & $-194 / A \pm m f F / A$ & $-9.18 \wedge \pm 9 / 1$ & ذوزئقه تحتائى & \\
\hline $.18 n^{2}$ & ( & $-\varepsilon r / q \pm \% q / r$ & $-q \mu / r \checkmark \pm r / 8$ & اركترو اسياينا & \\
\hline ATFY & .1818 & $-V \Delta / r \pm r r / \varepsilon$ & $-91 / 111 \pm 9 / 4$ & جناغى جنبرى بيستانى & \\
\hline . Nar & $\cdot M+1$ & $-\Lambda V / V \pm M T / \Lambda$ & $-V \Delta / / Y I \pm S / 1$ & ذوزنقه فوقانى & جֶ \\
\hline$\cdot \pi+4$ & $\cdot / m W$ & $-V V / \pm T V / \Lambda$ & $-11 r / / r m \pm V / 8$ & دندانهاى قدامى & \\
\hline $.1+W$ & - /AFA & $-r / \Delta \pm r V / 9$ & $-V / / \Lambda \pm V / r$ & ذوزنقه تحتانى & \\
\hline
\end{tabular}

مجله بيومكانيك ورنثـ 
جدول f. دامنه بايان فُعاليت عضلات طى هايان فاز اتكاي دويدن در دو كروه سالم و داراى عارضه سربهجلو

\begin{tabular}{|c|c|c|c|c|c|}
\hline \multirow{2}{*}{ اندازه اثر } & \multirow{2}{*}{ سطح معنى دارى } & \multicolumn{2}{|c|}{ ميانكين ثانحراف معيار } & \multirow{2}{*}{ عضله } & \multirow{2}{*}{ سمت } \\
\hline & & كروه سريهجلو & يزوه سالم & & \\
\hline .108 &.$/ T V$ & $-|V E / M| \pm Y / 9$ & $-m \Psi / / V \Delta \pm \Psi / \Delta$ & اركترو اسياينا & \\
\hline.$/ \pi q q$ & . IAFY & $-11 \Delta / 1 \Delta \Delta \pm q / r$ & $-\mid E T / R F \pm . / f$ & جناعي جنبرى يَستانى & \\
\hline.$/ P \Delta \Delta$ &.$/ M T$. & $-r \cdot V / A \pm 1 / r$ & $-Y A Q / Y R A \pm T / q$ & ذوزئقه فوقاثئي & | - ماسست \\
\hline .1 .04 & $.19 W$ & $-1 \Delta \& / 1 \Delta Y \pm \cdot / 9$ & $-\mid \Delta V / \Delta f \pm r / r$ & دندائهاي قدامى & \\
\hline . Larq &.$/ T V$ & $-1 \Delta \& / q r \pm . /$ & $-M R T / M T Y N q$ & ذوزيقه تحتثائى & \\
\hline .1 .14 &.$/ 94 A$ & $-M P V / M P \cdot \pm r / \Delta$ & $-r r e / / F \Delta \pm \Delta / F$ & اركترو أسياينا & \\
\hline$\cdot \mid 8 \cdot r$ &.$/ 4 I I$ & $-\mid \varepsilon r / r q \pm r / g$ & $-r \cdot e / \| 1 r \pm r / \Delta$ & جناغى جنبرى يَتانى & \\
\hline$. / r+r$ & $.19 \pi$ & $-r T q / V E \cdot \pm \cdot / 9$ & $-4|\cdot| r q \vee \pm 8 \mid$ & ذوزنقه فوقانى & جي \\
\hline 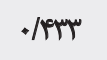 & / Atr & $-r q \Delta / F V t+/$ & $-U \Lambda T / r Y \pm r / \Lambda$ & دندانهاى قدامى & \\
\hline .1 .81 & .1911 & $-\mid r g / A+ \pm g / A$ & - IrV/F $\pm / V$ & ذوزنقه تحتانىى & \\
\hline
\end{tabular}

مجله بيومكانيك ورنش

$$
\begin{aligned}
& \text { معنى دارى وجود نداشت (ه> (P>). } \\
& \text { ث }
\end{aligned}
$$

هدف يُروهش حاضر بررسى الكوى زمانبندى عضلات تثنه و

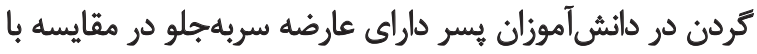

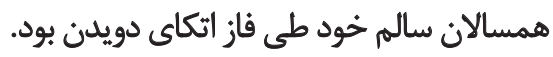
يافتههاى يروهش حاضر نشان داد كه در شروع و اتمام فعاليت

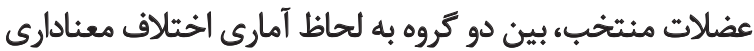
وجود ندارد. يك مطالعه بر روى زمان فراخوانى عضلات ناخيه لحاظ آماريه
ندارد (ه> P> P مقادير اندازه اثر در تمامي موارد در محدوده كوجى ثا متوسط بود (جدول شماره f). ملت زمان فعاليت عضلات اركترو اسياينا سمت راست

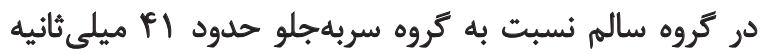

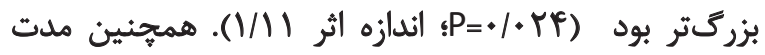

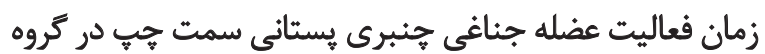

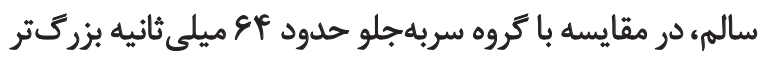

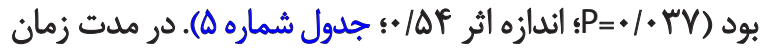

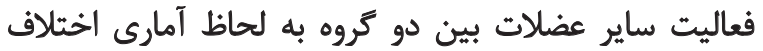

\begin{tabular}{|c|c|c|c|c|c|}
\hline \multirow{2}{*}{ اندازه اثر } & \multirow{2}{*}{ سطح معنى دارى } & \multicolumn{2}{|c|}{ ميانكين ثانحراف معيار } & \multirow{2}{*}{ عضله } & \multirow{2}{*}{ سمت } \\
\hline & & كروه سربه جلو & مروه سالم & & \\
\hline $1 / 1$ & $+/+\operatorname{me*}$ & Wret./r & WWFA +10 & اركترو أسياينا & \\
\hline +/VAq & $+1+\sqrt{9}$ & $V E / T H E V / T$ & $\| r / g r \pm 1 / 8$ & جناغى جنبرى يـستاني & \\
\hline$+/ M M$ & AfAT & $\mid Q H / T V \pm+/ 8$ & $r+v / r m+q / r$ & ذوزنقه فوقانى & راست \\
\hline ./TAF & . IAT. & IFT/IKrIT/ & $\| \varepsilon / V+ \pm 1 / r$ & دندائهاى قدامى & \\
\hline $1+4$ &.$/ 917$ & $r \Delta \cdot / r \& \Delta \pm N{ }^{\mu}$ & rgT/MNA土N & ذوزئقه تحتانىى & \\
\hline $.1 \cdot \lambda \mu$ & - /ATV & IW/YRE \pm Th & $10 Q / 18 A \pm F / T$ & إركترو أسيايثا & \\
\hline.$/ \Delta f \varepsilon$ & $.1 . r v^{*}$ & $A V / r \Delta \pm Q / T$ & $101 / 1.9 \pm \%$ & جناغى هنبرى يستانى & \\
\hline $.1 \cdot 1 r$ & $.19 W$ & $M F I / M F I \pm N q$ & $m e / m e r t q / 1$ & ذوزئقه فوقائى & 离 \\
\hline - mpa &.$/ D 1 Y$ & $r \mid \Delta / P \wedge r \pm f / f$ & $1 r \cdot 181 \pm r / 9$ & دنلائهاي قدامى & \\
\hline . /app & $\bullet / P \cdot V$ & $9 r / \Delta r \pm r \%$. & $\Delta V / Y Y \pm N Y$ & ذوزئقه تحتثائي & \\
\hline
\end{tabular}

جدول هـ ميزان فعاليت عضلات طي فاز انكاي طي دويدن در دو كروه سالمه و داراى عارضه سريهجلو

مجله بيومكانيك ورنش 


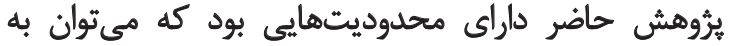

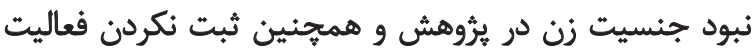

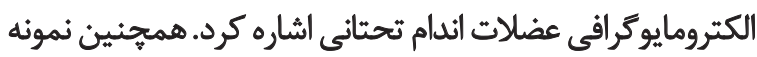

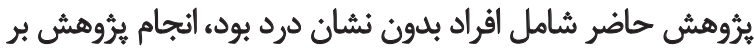

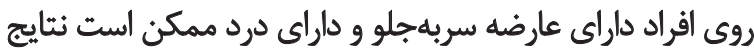

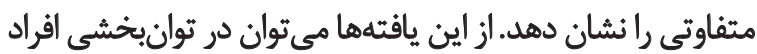
داراى عارضه سربهجلو در محيط هاى درمانى استفاده كرد.

مالاحظات اخلاقي

\section{ييروى از اصول اخلاق يثوهش}

همه اصول اخلاقى در اين مقاله رعايت شده است. شركت

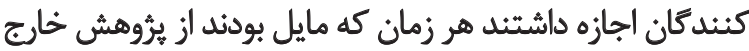

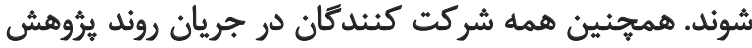

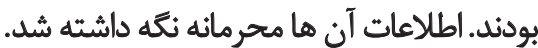

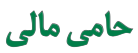

اين يُوهش هيجگكونه كمك مالى از سازمانيهاى دولتى، خصوصى و غيرانتفاعى دريافت نكرده است.

$$
\text { مشاركت نويسندكان }
$$

تمام نويسندكان در آمادهسازى اين مقاله مشاركت داشتهاند.

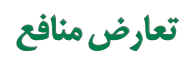

تمام نويسندكان در آمادهازى اين مقاله مشاركت داشتهاند.
شانه در بيماران مبتلا به درد كردن ايدئوياتيك در مقايسه با كروه

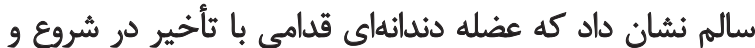

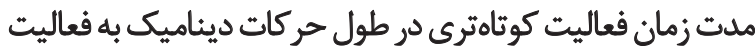

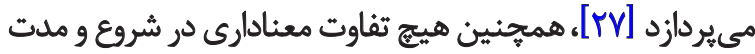

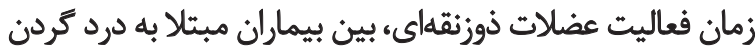

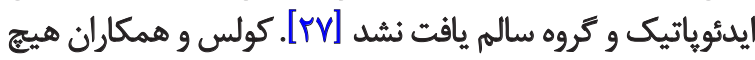

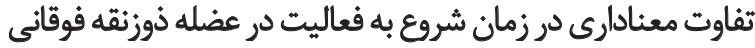

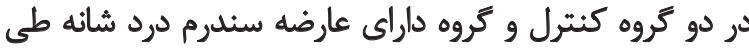

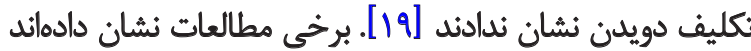

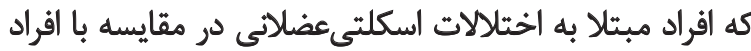

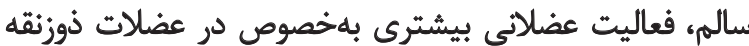

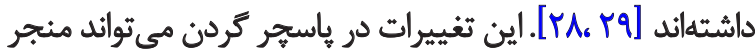

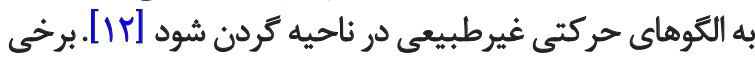

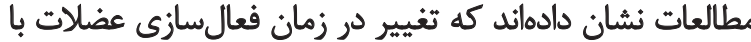

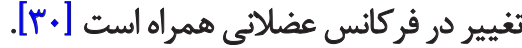
يافتههاى يرُوهش حاضر نشان داد كه مدت زمان فعاليت

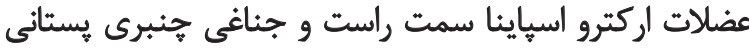

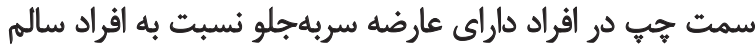

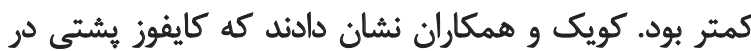

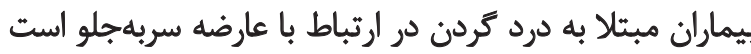

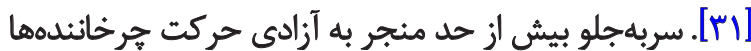

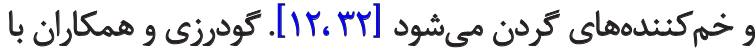

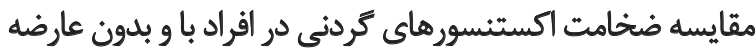

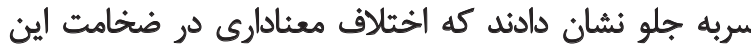

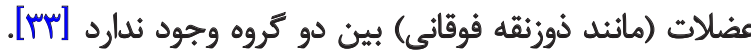

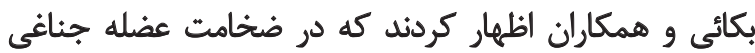

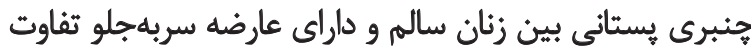

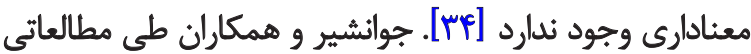

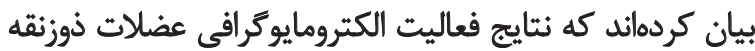

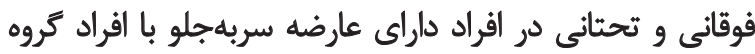

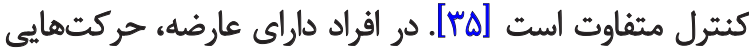

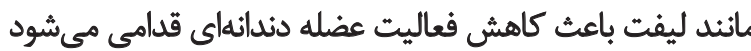

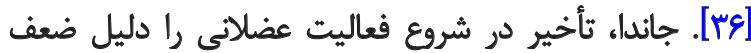

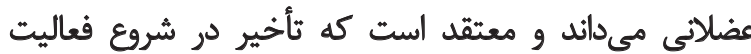

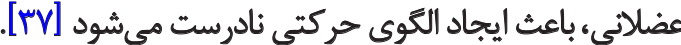

\section{نتيجهيَيرى نهايي}

شروع و يايان فعاليت منتخبى از عضلات ناحيه تنه و كردن

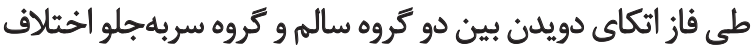

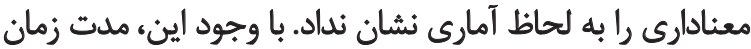

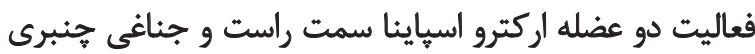

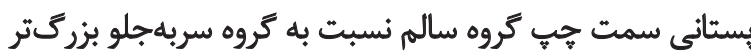

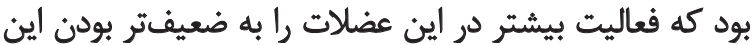




\section{References}

[1] Neumann DA. Kinesiology of the musculoskeletal system: Foundations for physical rehabilitation. St. Louis: Mosby; 2002

[2] Thigpen CA, Padua DA, Michener LA, Guskiewicz K, Giuliani C, Keene JD, et al. Head and shoulder posture affect scapular mechanics and muscle activity in overhead tasks. Journal of Electromyography and Kinesiology. 2010; 20(4):701-9. [DOI:10.1016/j.jelekin.2009.12.003] [PMID]

[3] Clarkson HM. Joint motion and function assessment: A research-based practical guide. Philadelphia: Lippincott Williams \& Wilkins; 2005.

[4] Griegel-Morris P, Larson K, Mueller-Klaus K, Oatis CA. Incidence of common postural abnormalities in the cervical, shoulder, and thoracic regions and their association with pain in two age groups of healthy subjects. Physical Therapy. 1992; 72(6):425-31. [DOI:10.1093/ptj/72.6.425] [PMID]

[5] Williams R. Heads up on more reason your back hurts. Sports Medicine. 2006; 8(2):119-22.

[6] Peterson-Kendall F, Kendall-McCre Provance $P$, McIntyre-Rodgers $M$ Romani W. Muscles testing and function with posture and pain. Philadelphia: Lippincott Williams \& Wilkins; 2005.

[7] Yip $\mathrm{CH}$, Chiu TT, Poon AT. The relationship between head posture and severity and disability of patients with neck pain. Manual Therapy. 2008; 13(2):148-54. [DOI:10.1016/j.math.2006.11.002] [PMID]

[8] Kamper SJ, Henschke N, Hestbaek L, Dunn KM, Williams CM. Musculoskeletal pain in children and adolescents. Brazilian Journal of Physical Therapy. 2016; 20(3):275-84. [DOI:10.1590/bjpt-rbf.2014.0149] [PMID] [PMCID]

[9] Ishida H, Suehiro T, Kurozumi C, Ono K, Ando S, Watanabe S. Correlation between neck slope angle and deep cervical flexor muscle thickness in healthy participants. Journal of Bodywork and Movement Therapies. 2015; 19(4):717-21. [DOI:10.1016/j.jbmt.2015.04.001] [PMID]

[10] Harman K, Hubley-Kozey CL, Butler H. Effectiveness of an exercise program to improve forward head posture in normal adults: A randomized, controlled 10-week trial. Journal of Manual \& Manipulative Therapy. 2005; 13(3):163-76. [DOI:10.1179/106698105790824888]

[11] Lynch SS, Thigpen CA, Mihalik JP, Prentice WE, Padua D. The effects of an exercise intervention on forward head and rounded shoulder postures in elite swimmers. British Journal of Sports Medicine. 2010 44(5):376-81. [DOI:10.1136/bjsm.2009.066837] [PMID]

[12] Yoo WG, An DH. The relationship between the active cervical range of motion and changes in head and neck posture after continuous VDT work. Industrial Health. 2009; 47(2):183-8. [DOI:10.2486/inde health.47.183] [PMID]

[13] Paillard T. Effects of general and local fatigue on postural control: A review. Neuroscience \& Biobehavioral Reviews. 2012; 36(1):162-76. [DOI:10.1016/j.neubiorev.2011.05.009] [PMID]

[14] Silva AG, Sharples $P$, Johnson MI. Studies comparing surrogate measures for head posture in individuals with and without neck pain Physical Therapy Reviews. 2010; 15(1):12-22. [DOI:10.1179/17432881 0X12647087218631]

[15] Edmondston SJ, Sharp M, Symes A, Alhabib N, Allison GT. Changes in mechanical load and extensor muscle activity in the cervico-thoracic spine induced by sitting posture modification. Ergonomics. 2011 54(2):179-86. [DOI:10.1080/00140139.2010.544765] [PMID]

[16] Schulte E, Kallenberg L, Christensen H, Disselhorst-Klug C, Hermens HJ, Rau G, et al. Comparison of the electromyographic activity in the upper trapezius and biceps brachii muscle in subjects with muscular disorders: A pilot study. European Journal of Applied Physiology. 2006; 96(2):185 93. [DOI:10.1007/s00421-004-1291-2] [PMID]

[17] Hodges PW, Richardson CA. Altered trunk muscle recruitment in people with low back pain with upper limb movement at different speeds. Archives of Physical Medicine and Rehabilitation. 1999; 80(9):1005-12. [DOI:10.1016/S0003-9993(99)90052-7]

[18] O'Sullivan P, Twomey L, Allison G, Sinclair J, Miller K, Knox J. Altered patterns of abdominal muscle activation in patients with chronic low back pain. Australian Journal of Physiotherapy. 1997; 43(2):91-8. [DOI:10.1016/S0004-9514(14)60403-7]

[19] Cools AM, Witvrouw EE, Declercq GA, Danneels LA, Cambier DC. Scapular muscle recruitment patterns: Trapezius muscle latency with and without impingement symptoms. The American Journal of Sports Medicine. 2003; 31(4):542-9. [DOI:10.1177/03635465030310041101] [PMID]

[20] Moraes GF, Faria CD, Teixeira-Salmela LF. Scapular muscle recruitment patterns and isokinetic strength ratios of the shoulder rotator muscles in individuals with and without impingement syndrome. Journal of Shoulder and Elbow Surgery. 2008; 17(1):S48-S53. [DOI:10.1016/j. jse.2007.08.007] [PMID]

[21] Faul F, Erdfelder E, Lang AG, Buchner A. G*Power 3: A flexible statistical power analysis program for the social, behavioral, and biomedical sciences. Behavior Research Methods. 2007; 39(2):175-91. [DOI:10.3758/ BF03193146] [PMID]

[22] Salahzadeh Z, Maroufi N, Ahmadi A, Behtash H, Razmjoo A, Gohar $M$, et al. Assessment of forward head posture in females: Observational and photogrammetry methods. Journal of Back and Musculoskeletal Rehabilitation. 2014; 27(2):131-9. [DOI:10.3233/BMR-130426] [PMID]

[23] Winter DA. Biomechanics and motor control of human movement Hoboken: John Wiley \& Sons; 2009. [DOI:10.1002/9780470549148]

[24] Falla D, Jull G, Hodges P. Feedforward activity of the cervical flexor muscles during voluntary arm movements is delayed in chronic neck pain. Experimental Brain Research. 2004; 157(1):43-8. [DOI:10.1007/ s00221-003-1814-9] [PMID]

[25] Hermens HJ, Hutten MM. Muscle activation in chronic pain: its treatment using a new approach of myofeedback. International Journal of Industrial Ergonomics. 2002; 30(4):325-36. [DOI:10.1016/S01698141(02)00134-8]

[26] Murley GS, Menz HB, Landorf KB. Foot posture influences the electromyographic activity of selected lower limb muscles during gait. Journa of Foot and Ankle Research. 2009; 2:35. [DOI:10.1186/1757-1146-2-35] [PMID] [PMCID]

[27] Helgadottir $\mathrm{H}$, Kristjansson E, Einarsson E, Karduna A, Jonsson H. Altered activity of the serratus anterior during unilateral arm elevation in patients with cervical disorders. Journal of Electromyography and Kinesiology. 2011; 21(6):947-53. [DOI:10.1016/j.jelekin.2011.07.007] [PMID]

[28] Veiersted KB, Westgaard RH, Andersen P. Electromyographic evaluation of muscular work pattern as a predictor of trapezius myalgia. Scandinavian Journal of Work, Environment \& Health. 1993; 19(4):284-90. [DOI:10.5271/sjweh.1472] [PMID]

[29] Veiersted K, Westgaard R, Andersen P. Pattern of muscle activity during stereotyped work and its relation to muscle pain. International $\mathrm{Ar}$ chives of Occupational and Environmental Health. 1990; 62(1):31-41. [DOI:10.1007/BF00397846] [PMID] 
[30] Farley CT, Gonzalez O. Leg stiffness and stride frequency in human running. Journal of Biomechanics. 1996; 29(2):181-6. [DOI:10.1016/00219290(95)00029-1]

[31] Quek J, Pua YH, Clark RA, Bryant AL. Effects of thoracic kyphosis and forward head posture on cervical range of motion in older adults. Manual Therapy. 2013; 18(1):65-71. [DOI:10.1016/j.math.2012.07.005] [PMID]

[32] Ro H, Gong W, Ma S. Correlations between and absolute rotation angle, anterior weight bearing, range of flexion, and extension motion in cervical herniated nucleus pulposus. Journal of Physical Therapy Science. 2010; 22(4):447-50. [DOI:10.1589/jpts.22.447]

[33] Goodarzi F, Karimi N, Rahnama L, Khodakarim S. Differences in cervical extensor muscles thickness on subjects with normal head posture and forward head posture; an ultrasonography study. Journal of Rehabilitation Sciences and Research. 2015; 2(2):23-6. [DOI:10.30476/ JRSR.2015.41069]

[34] Bokaee F, Rezasoltani A, Manshadi FD, Naimi SS, Baghban AA, Azimi $\mathrm{H}$. Comparison of cervical muscle thickness between asymptomatic women with and without forward head posture. Brazilian Journal of Physical Therapy. 2017; 21(3):206-11. [DOI:10.1016/j.bjpt.2017.04.003] [PMID] [PMCID]

[35] Javanshir Kh, Mohseni-Bandpei MA, Amiri M, Rezasoltani A, Rahgozar $M$. [The comparison of Longus colli muscle size and shape ratio between healthy subjects and chronic neck pain patients using ultrasonography (Persian)]. J Gorgan Univ Med Sci. 2010; 12(1):33-7.

[36] Weon JH, Oh JS, Cynn HS, Kim YW, Kwon OY, Yi CH. Influence of forward head posture on scapular upward rotators during isometric shoulder flexion. Journal of Bodywork and Movement Therapies. 2010; 14(4):367-74. [DOI:10.1016/j.jbmt.2009.06.006] [PMID]

[37] Janda V. Muscle weakness and inhibition (pseudoparesis) in back pain syndromes. In: Grieve GP, editor. Modern Manual Therapy of the Vertebral Column. London: Churchill-Livingstone; 1986 
This Page Intentionally Left Blank 